\title{
1 A Possible Inductive Mechanism for Magnetogenetics
}

2

3 Dr. Michael G. Christiansen, ${ }^{1, *}$ William Hornslien, ${ }^{2}$ Prof. Simone Schuerle ${ }^{1, *}$

$4 \quad 1$ ETH Zürich, Department of Health Sciences and Technology

52 Norwegian University of Science and Technology, Department of Mathematical Sciences

$6 *$ Authors to whom correspondence should be addressed: $<$ michael.christiansen@hest.ethz.ch $>$ and

7 <simone.schuerle@hest.ethz.ch>

\section{Abstract}

Reports of genetically conferred sensitivity to magnetic stimuli have preceded plausible mechanistic explanations. Frequently, these experiments rely on a fusion of ferritin with a transient receptor potential vanniloid channel protein, speculating associated mechanical or thermal cues. However, it has been argued compellingly that the small magnetic moment of ferritin precludes these possibilities. Here, we offer an alternative hypothesis based on stochastic resonance that does not require appreciable interaction of ferritin with the applied field. Rather, we suggest that ferritin might act merely as a localized source of high frequency inductive noise on the membrane. When combined with externally applied time-varying fields, this noise might help surmount the activation threshold of endogenous voltage-gated ion channels. To explore this concept, we use the stochastic Landau-Lifshitz-Gilbert equation to model magnetization dynamics and compare the magnetic field noise resulting from ferritin and from a $15 \mathrm{~nm}$ magnetite particle. 


\section{$1 \quad$ Introduction}

The broadly recognized usefulness of optogenetics and chemogenetics for enabling targeted actuation of neurons has spurred interest in development of an analogous "magnetogenetic" technique. Triggering activity via wireless, invisible, and facile magnetic stimuli in transfected neuronal subpopulations holds obvious appeal, and reports describing conferred sensitivity to magnetic fields have already appeared (Stanley et al., 2012; Stanley et al., 2016; Stanley, Sauer, Kane, Dordick, \& Friedman, 2015; Wheeler et al., 2016). These studies typically incorporate one or more units of the iron storage protein ferritin into a transient receptor potential vanniloid (TRPV) channel protein. Because the TRPV family is known to trigger calcium influx in response to mechanical and thermal cues, magnetic interactions with ferritin have been presumed to be a source of similar effects (Barbic, 2019). However, cogent critique has cast doubt on these speculated mechanisms (Meister, 2016). The interaction of ferritin with attainable magnetic fields is far too weak for mechanical actuation, hysteresis heating of ferritin is demonstrably negligible, and even if extraordinary heat flow were to occur, local temperature increase is not expected (Anikeeva \& Jasanoff, 2016; Davis et al., 2020; Keblinski, Cahill, Bodapati, Sullivan, \& Taton, 2006). With an identified and tested mechanism, magnetogenetics could become a tool that can be used confidently and further optimized.

To help bridge this explanatory gap, here we suggest a potential mechanism applicable to excitable cell types expressing voltage gated ion channels. It accounts for actuation with inductively coupled stochastic resonance, mediated by ferritin localized on the cell membrane. Counterintuitively, ferritin may actually be better suited to this purpose than larger magnetite particles. To show this, we consider how the interaction of ferritin with a neighboring voltage-gated ion channel, in combination with a time-varying magnetic field, could plausibly serve to open these channels. We identify experiments that could test this hypothesis and consider possible implications if it were found to be valid.

\section{Results and Discussion}

\section{Inductively Coupled Stochastic Resonance}

A realistic model of ferritin is an essential starting point for any plausible mechanistic explanation of magnetogenetics. Historically, both the reported magnetic properties of ferritin and their interpretations have varied considerably, depending on their biological source and sample preparation. Nevertheless, magnetic characterization of ferritin spans nearly eight decades and can at least inform reasonable bounds for expected behavior (Michaelis, Coryell, \& Granick, 1943). Ferritin consists of a protein shell with an outer diameter of approximately $12 \mathrm{~nm}$ that surrounds a biomineralized core with diameter 5.5 to $6.0 \mathrm{~nm}$ in humans, ranging up to about $8 \mathrm{~nm}$ in mollusks (Chasteen \& Harrison, 1999). The size and crystallinity of human ferritin is comparable to ferritin derived from horse spleens, which is often studied. The core consists primarily of ferrihydrite with an approximate stoichiometry 
$5 \mathrm{Fe}_{2} \mathrm{O}_{3} \cdot 9 \mathrm{H}_{2} \mathrm{O}$, possibly incorporating trace phosphate impurities (Jutz, van Rijn, Santos Miranda, \& Böker, 2015). Whether attributable to uncompensated antiferromagnetically ordered spins or the existence of multiple phases (Cowley, Janney, Gerkin, \& Buseck, 2000), multiple empirical sources contend that ferritin's ferrihydrite core is superparamagnetic at physiological temperatures with a weak magnetic moment of approximately $300 \mu_{\mathrm{B}}$ (Brooks, Vymazal, Goldfarb, Bulte, \& Aisen, 1998; Kilcoyne \& Cywinski, 1995; Makhlouf, Parker, \& Berkowitz, 1997). Experimental evidence for both an influence on T2 relaxation and feasibility of magnetic cell separation for bacteria expressing ferritin offer support for applying this interpretation to transgenically expressed ferritin (Liu et al., 2016; Matsumoto, Chen, Anikeeva, \& Jasanoff, 2015).

In the hypothesis offered here, we suggest a mechanistic role for this superparamagnetism that shifts focus away from the thermal and mechanical sensitivities that have dominated the discussion and instead consider interaction with voltage gated ion channels (Figure 1a). Although voltage responsiveness has been observed in the TRPV family, interaction with endogenous ion channels gated at lower voltages could be more relevant (Nilius et al., 2005). A recent response from Wheeler et al. stated that a high density of expressed magnetogenetic membrane channels is required to reproduce their experimental results (Wheeler, Deppmann, Patel, \& Güler, 2019). While this might be interpreted to reflect a low likelihood of an individual channel opening in response to magnetic stimulation, it would also be consistent with a proximity-based effect, in which the increased chances of adjacent ferritin on the membrane contributes to the mechanism. As the density of expressed proteins increases, even relatively rare or transient situations such as a ferritin sitting directly adjacent to a voltage-gated ion channel can be reasonably considered (Figure 1a).

Approximating the moment of ferritin as a $300 \mu_{\mathrm{B}}$ point dipole, the expected field magnitude at its surface is only a few $\mathrm{mT}$ and drops to a magnitude comparable to the geomagnetic field within 25 $\mathrm{nm}$ (Figure 1b). Assuming a voltage-gated ion channel with approximately $10 \mathrm{~nm}$ diameter, a ferritin directly adjacent to it should produce at most a small field of about $0.4 \mathrm{mT}$ in the center of the channel. While this field magnitude is minute, inductive effects are determined by $d B / d t$, and the timescale of fluctuation of the moment plays a role of equal importance in determining this quantity. Individual superparamagnetic fluctuation of magnetic moments between preferred axes at a timescale kinetically limited by their anisotropy barrier is a well studied and directly observed phenomenon (Wernsdorfer et al., 1997). The temperature dependence of the timescale of stochastic reversal $\tau$ can be described in terms of an attempt rate $\tau_{0}$, an intrinsic energy barrier $U_{B}$, the Boltzmann constant $k_{B}$, and the absolute temperature $T$ (Néel, 1949):

$$
\tau=\tau_{0} \operatorname{Exp}\left(\frac{U_{B}}{k_{B} T}\right)
$$

The key relevant input quantities, $\tau_{0}$ and $U_{B}$, can be estimated from data collected on various ferritin preparations. In the interest of putting the proposed mechanism on firmer footing, values can be selected 
1 from a reasonable range that tend to bound or mitigate the relevant effects. $\tau_{0}$ for superparamagnetic

2 particles typically ranges from 100 fs to $1 \mathrm{~ns}$ (Kilcoyne \& Cywinski, 1995), and although values on the

3 order of 1 to 10 ps have been fitted for ferritin (Dickson et al., 1993; Kilcoyne \& Cywinski, 1995), here

4 we conservatively take $1 \mathrm{~ns}$. Reported blocking temperatures seem to depend on preparation and

5 characterization method. Although a blocking temperature of about $12 \mathrm{~K}$ seems most likely and is

6 consistent with low temperature magnetization measurements (Dickson et al., 1993; Kilcoyne \&

7 Cywinski, 1995; Makhlouf et al., 1997), we have assumed the higher reported value of $40 \mathrm{~K}$ because

8 increased anisotropy should also tend to mitigate the hypothesized effect (Chasteen \& Harrison, 1999).

a)

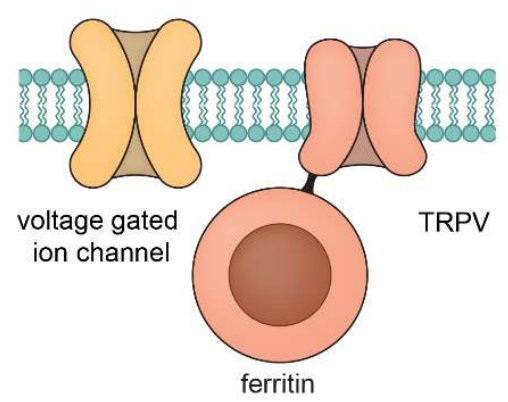

d) Biphasic system (channel)

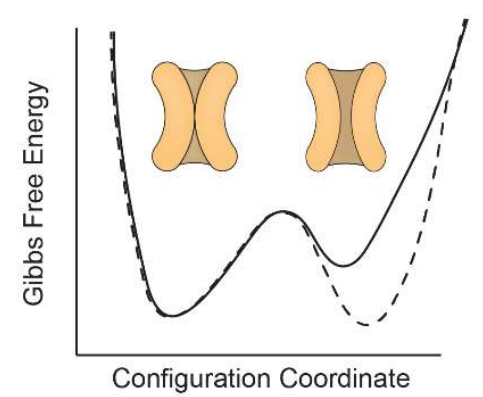

b)

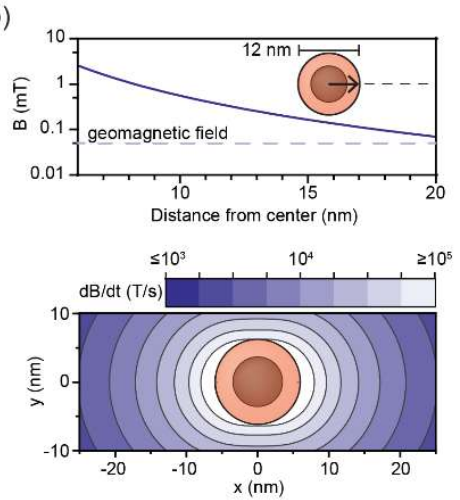

Subthreshold Stimulus

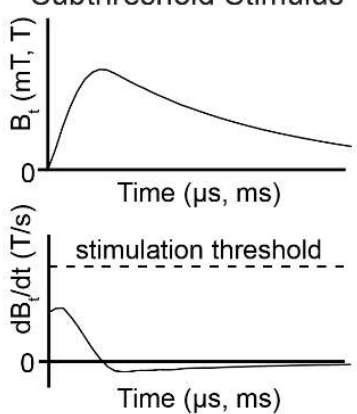

c) Normal dB/dt $A B$

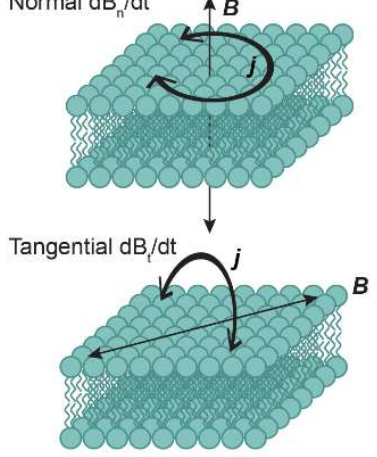

Noise source

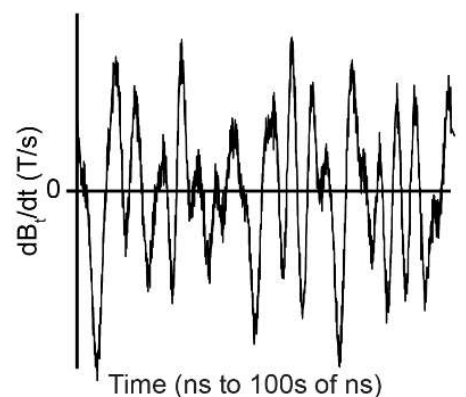

Figure 1. Conceptual illustrations. a) A transgenic TRPV channel protein incorporating a ferritin unit is expressed in the membrane, perhaps occurring near to any one of a variety of voltage-gated ion channels endogenously present in excitable cells. b) At top, the expected field magnitude versus distance is shown assuming a moment of $300 \mu \mathrm{B}$ for ferritin. At bottom, the rate of change of the field is mapped for a macrospin model with conservative assumptions for kinetically limited reversal. c) A schematic illustrates why the component of the field changing tangentially to the membrane is most relevant to transmembrane potentials. d) The components that might enable inductively coupled stochastic resonance are represented pictorially. At left, the voltage gated ion channel represents a biphasic system that responds to applied potentials, including those induced by time varying fields. In center, a subthreshold stimulus is provided by an externally applied time-varying field. At right, noise is supplied by the fluctuation or precession of one or more ferritin magnetic moments.

As shown in the supplementary materials, these assumptions bound the fluctuation of ferritin to a characteristic timescale of about 20 ns. Time variation in magnetic flux on the channel occurring tangentially to the membrane would induce transmembrane voltage noise (Figure 1c). Estimating the order of magnitude of this $d B / d t$ noise at the center of the ion channel, we find: 


$$
\frac{d B}{d t} \approx \frac{\Delta B}{\Delta \tau}=\frac{2(0.0004 \mathrm{~T})}{2 \times 10^{-8} \mathrm{~s}}=4 \times 10^{4} \mathrm{~T} \mathrm{~s}^{-1}
$$

This bound for $d B / d t$ in the local vicinity of the channel is comparable to empirically determined thresholds for inductive stimulation of neurons, reported to be about $2 \times 10^{3} \mathrm{~T} \mathrm{~s}^{-1}$ (McRobbie \& Foster, 1984). The high frequency and limited spatial extent of this noise ensure that alone it should not produce spontaneous stimulation. Rather, the presence of one or more ferritins could merely act as a source of localized inductive noise on the membrane and ion channels.

This situation is altered by introducing a time varying magnetic stimulus acting on a timescale relevant to the channel dynamics, perhaps ranging from $10 \mathrm{~s}$ of $\mu$ s to $100 \mathrm{~s}$ of $\mathrm{ms}$. In their recent response to a failed replication study, Wheeler et al. stress the necessity for a time varying magnetic stimulus for reproducing their results (Wheeler et al., 2019). Similarly, other reports involving ferritin fused to TRPV often employ alternating magnetic fields in the 100s of kHz (Stanley et al., 2012; Stanley et al., 2015). Time-varying fields can elicit widespread induced voltages on the membranes of exposed neurons, a principle relied upon for transcranial magnetic stimulation (Romero, Davare, Armendariz, \& Janssen, 2019). In the absence of magnetogenetic expression, these voltages are likely to be well below the necessary threshold to trigger an action potential.

With a nearby ferritin, however, a stochastic resonance phenomenon might emerge that accounts for the opening of the channel (McDonnell \& Abbott, 2009; McNamara \& Wiesenfeld, 1989). The necessary components are present: 1) The conformation of the voltage gated channels as a biphasic system, 2) the externally applied $d B / d t$ as a subthreshold perturbation, and 3) ferritin as a source of high frequency $d B / d t$ noise with a magnitude relevant to the typical threshold of actuation (Figure 1d). The most appealing feature of stochastic resonance in this case is that it does not require ferritin to interact with the applied magnetic field at a scale comparable to $k_{B} T$. Rather, the voltage induced by the applied magnetic stimulus itself could supply energy for reconfiguring the channel, and the noise generated by ferritin could merely aid in surmounting the kinetic barrier setting the threshold of actuation.

\section{Modelling Magnetic Fluctuation and Precession in Ferritin and Comparing to Magnetite}

An intriguing feature of this hypothetical mechanism is its implication that ferritin may have features that make it better suited to magnetogenetic stimulation than magnetic nanoparticles with large magnetic moments. As illustrative examples, we have selected ferritin and a $15 \mathrm{~nm}$ magnetite particle with a $2.5 \mathrm{~nm}$ polymer or silica shell. Using the stochastic Landau-Lifshitz-Gilbert (sLLG) equation to describe both precession and the influence of thermal agitation (Figure 2a), we model the dynamical behavior of a single particle and infer tangential $B$ at various locations near the particle (Figure $\mathbf{2 b}$ ) (Brown, 1963; Usov, 2010). Notably, this noisy signal has an additional high frequency contribution 
1 from precession, and a low pass filter reveals behavior arising from kinetically limited reversal. Full

2 details of this simple model can be found in the supplementary materials.

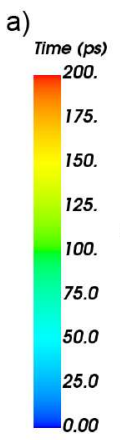

Stochastic LLG Model

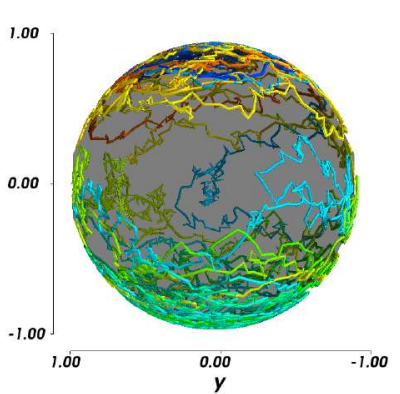

b) Spatial Variation
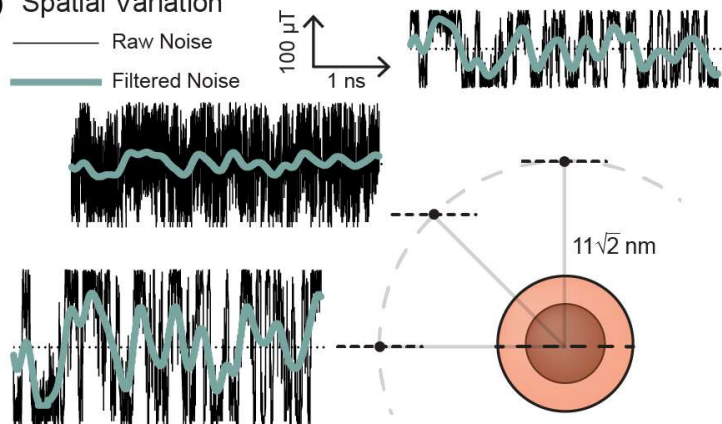

c)

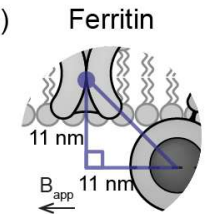

$15 \mathrm{~nm}$ Magnetite Nanoparticle
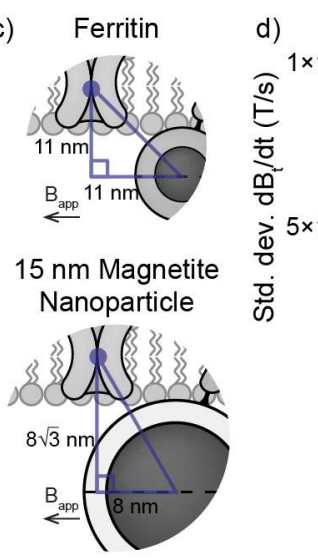
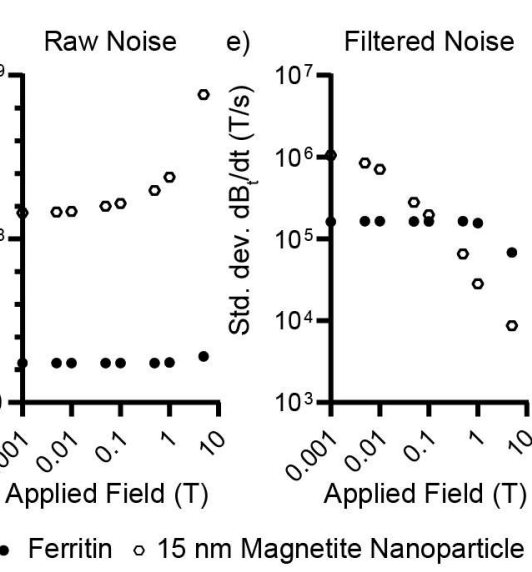

f) Additive Effects

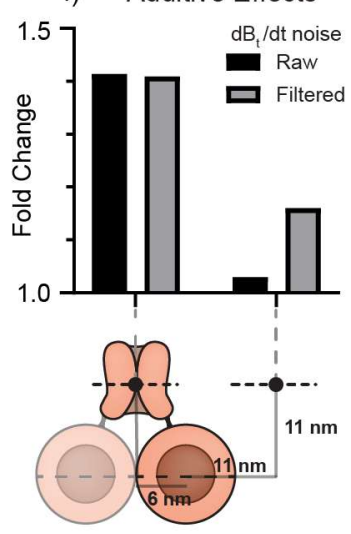

Figure 2. Simulations of noise produced by ferritin and their comparison to a $15 \mathrm{~nm}$ magnetite particle. a) $200 \mathrm{ps}$ of the time evolution of the orientation of the magnetic moment of ferritin simulated with a stochastic Landau-Lifshitz-Gilbert (sLLG) model. Plot created with Mayavi in Python (Ramachandran \& Varoquaux, 2011). b) Tangential projection of magnetic field versus time at three positions equidistant $(15.6 \mathrm{~nm})$ from a simulated ferritin. A low pass filter set at $10 \mathrm{GHz}$ reveals signal resulting from stochastic reversal. The dashed line atop the ferritin shows the orientation of its easy axis. c) Simplified geometric assumptions for calculating the component of the field tangential to the membrane are shown explicitly for both a ferritin and the $15 \mathrm{~nm}$ magnetite particle. d) Standard deviation of tangential $d B / d t$ noise for simulations of $6 \times 10^{6}$ steps each are shown for a range of applied quasimagnetostatic fields. The raw noise is dominated by the influence of precession. e) A low pass filter set at $10 \mathrm{GHz}$ for ferritin and $1 \mathrm{GHz}$ for magnetite was applied to the same noise data represented in panel $\mathrm{d}$ ), and tangential $d B / d t$ noise was calculated to show the applied field dependence of noise originating from stochastic reversal. f) The additive effects of noise from two adjacent non-interacting ferritins (bottom) is considered. The fold increase in the standard deviation of the noise signal is shown for two points. The first is at the center of the membrane and equidistant from the two ferritins and the other assumes similar geometry to panel c.)

Simplified geometric assumptions used for predicting tangential $d B / d t$ noise are depicted in

Figure $2 \mathbf{c}$ for both ferritin and magnetite. Since the concept of inductively coupled stochastic resonance relies upon noise sources that persist despite the external application of quasistatic fields, we considered the influence of an applied magnetic field on fluctuation. The contribution to tangential $d B / d t$ noise arising from precession remains approximately constant or increases at sufficiently high magnitudes, as shown in Figure 2d. In contrast, the component arising from kinetically limited reversal is ultimately suppressed by high magnitude fields, as shown in Figure 2e. 
Despite possessing a moment nearly 300 times weaker than the $15 \mathrm{~nm}$ magnetite particle, this

\section{Experiments to Test this Hypothesis}

Although we have endeavored to frame this hypothesis as realistically as possible, we emphasize the need for further inquiry and direct experimental evidence. In this spirit, we suggest several ideas for experiments that could test various aspects.

1. The idea that ferritin is acting on voltage-gated ion channels rather than TRPV is directly testable by expressing some geometrically similar impermeable transmembrane protein incorporating ferritin in similar manner. If magnetogenetic stimulation were still observed, it would strongly suggest the relevance of interactions between ferritin and endogenous proteins. The extent of expression is clearly an important variable to consider in such an experiment. Magnetogenetically transfected neurons could serve as a positive control and wildtype neurons could serve as a negative control.

2. Applying a uniform pulsed field to in vitro samples using capacitive discharge into a suitable field coil would allow external $d B / d t$ stimuli to be applied measurably and controllably. Observing GCaMP fluorescence to monitor neuronal response, the endogenous threshold of wild type inductive actuation could be determined. By comparing to neurons expressing abundant magnetogenetic proteins, this experiment could measure the extent to which the $d B / d t$ threshold is lowered upon transfection.

3. To probe the mechanistic role of ferritin, it might be feasible to influence its dynamics with fields applied simultaneously with a $d B / d t$ stimulus. If the frequency components of the 

noise arising from stochastic reversal are ultimately most important, then a superimposed magnetostatic field of sufficient magnitude (greater than $2 \mathrm{~T}$, Figure 2e) might be used to suppress inductive noise. If noise arising from precession is more important, the system could instead be driven with a microwave frequency electromagnetic field corresponding to the ferromagnetic resonance frequency of the ferritin. If either of these approaches resulted in quantifiable changes in the $d B / d t$ threshold required for magnetogentic actuation, it would not be well explained by other proposed mechanisms (Barbic, 2019).

\section{Conclusion}

Inductively coupled stochastic resonance as a mechanism for stimulation is appealingly consistent not only with the features of some reported magnetogenetic experiments, but also with the theoretical critiques they have provoked. A few potentially important conceptual issues are left unexamined here, such as identifying which voltage gated ion channels are best suited to this form of stimulation or predicting spatial distributions of induced voltages. This hypothesis is limited to schemes employing ferritin fusions and time-varying magnetic stimuli, and offers limited or no insight into natural magnetoreception or reported magnetogenetic stimulation with constant fields.*

If experimental evidence were to ultimately support and refine this concept, the implications could be considerable. Most obviously, it would provide a path for rational design and optimization of magnetogenetic techniques. Additionally, time-varying fields supplied through capacitive discharge at amplitudes far below those used in transcranial magnetic stimulation could offer a low power, straightforward, and scalable approach to field generation. Such magnetic field stimuli might even be supplied by wearable devices, a consideration that would become relevant if magnetogenetics were to ultimately serve as a therapeutic technology.

\section{Acknowledgements}

M.G.C. is supported by an ETH Zurich Postdoctoral Fellowship. S.S. is supported by the Branco Weiss Fellowship of the Society in Science.

\section{Competing interests}

None to declare.

\footnotetext{
* Although natural occurrences such as lightning strikes can generate strong transient magnetic fields, the evolutionary context of natural magnetoreception seems to be centered on navigation in the weak geomagnetic field.
} 


\section{References}

Anikeeva, P., \& Jasanoff, A. (2016). Problems on the back of an envelope. eLife, 5, e19569. doi:10.7554/eLife.19569

Barbic, M. (2019). Possible magneto-mechanical and magneto-thermal mechanisms of ion channel activation in magnetogenetics. eLife, 8, e45807. doi:10.7554/eLife.45807

Brooks, R. A., Vymazal, J., Goldfarb, R. B., Bulte, J. W. M., \& Aisen, P. (1998). Relaxometry and magnetometry of ferritin. Magnetic Resonance in Medicine, 40(2), 227-235. doi:10.1002/mrm. 1910400208

Brown, W. F. (1963). Thermal Fluctuations of a Single-Domain Particle. Physical Review, 130(5), $1677-$ 1686. doi:10.1103/PhysRev.130.1677

Chasteen, N. D., \& Harrison, P. M. (1999). Mineralization in ferritin: an efficient means of iron storage. Journal of structural biology, 126(3), 182-194.

Cowley, J. M., Janney, D. E., Gerkin, R. C., \& Buseck, P. R. (2000). The Structure of Ferritin Cores Determined by Electron Nanodiffraction. Journal of structural biology, 131(3), 210-216. doi:https://doi.org/10.1006/jsbi.2000.4292

Davis, H. C., Kang, S., Lee, J.-H., Shin, T.-H., Putterman, H., Cheon, J., \& Shapiro, M. G. (2020). Nanoscale Heat Transfer from Magnetic Nanoparticles and Ferritin in an Alternating Magnetic Field. Biophysical Journal, 118(6), 1502-1510. doi:https://doi.org/10.1016/j.bpj.2020.01.028

Dickson, D. P. E., Reid, N. M. K., Hunt, C., Williams, H. D., El-Hilo, M., \& O'Grady, K. (1993). Determination of $\mathrm{f} 0$ for fine magnetic particles. Journal of Magnetism and Magnetic Materials, 125(3), 345-350. doi:https://doi.org/10.1016/0304-8853(93)90109-F

Jutz, G., van Rijn, P., Santos Miranda, B., \& Böker, A. (2015). Ferritin: A Versatile Building Block for Bionanotechnology. Chemical Reviews, 115(4), 1653-1701. doi:10.1021/cr400011b

Keblinski, P., Cahill, D. G., Bodapati, A., Sullivan, C. R., \& Taton, T. A. (2006). Limits of localized heating by electromagnetically excited nanoparticles. Journal of Applied Physics, 100(5), 054305. doi:10.1063/1.2335783

Kilcoyne, S. H., \& Cywinski, R. (1995). Ferritin: a model superparamagnet. Journal of Magnetism and Magnetic Materials, 140-144, 1466-1467. doi:https://doi.org/10.1016/0304-8853(94)00626-1

Liu, X., Lopez, P. A., Giessen, T. W., Giles, M., Way, J. C., \& Silver, P. A. (2016). Engineering Genetically-Encoded Mineralization and Magnetism via Directed Evolution. Scientific Reports, 6(1), 38019. doi:10.1038/srep38019

Makhlouf, S. A., Parker, F. T., \& Berkowitz, A. E. (1997). Magnetic hysteresis anomalies in ferritin. Physical Review B, 55(22), R14717-R14720. doi:10.1103/PhysRevB.55.R14717

Matsumoto, Y., Chen, R., Anikeeva, P., \& Jasanoff, A. (2015). Engineering intracellular biomineralization and biosensing by a magnetic protein. Nature Communications, 6(1), 8721. doi: $10.1038 /$ ncomms 9721

McDonnell, M. D., \& Abbott, D. (2009). What Is Stochastic Resonance? Definitions, Misconceptions, Debates, and Its Relevance to Biology. PLOS Computational Biology, 5(5), e1000348. doi:10.1371/journal.pcbi.1000348

McNamara, B., \& Wiesenfeld, K. (1989). Theory of stochastic resonance. Physical Review A, 39(9), 4854-4869. doi:10.1103/PhysRevA.39.4854

McRobbie, D., \& Foster, M. A. (1984). Thresholds for biological effects of time-varying magnetic fields. Clinical Physics and Physiological Measurement, 5(2), 67-78. doi:10.1088/01430815/5/2/002

Meister, M. (2016). Physical limits to magnetogenetics. eLife, 5, e17210. doi:10.7554/eLife.17210

Michaelis, L., Coryell, C. D., \& Granick, S. (1943). Ferritin III. The magnetic properties of ferritin and some other colloidal ferric compounds. Journal of Biological Chemistry, 148(3), 463-480.

Néel, L. (1949). Théorie du traînage magnétique des ferromagnétiques en grains fins avec applications aux terres cuites. Ann. géophys., 5, 99-136.

Nilius, B., Talavera, K., Owsianik, G., Prenen, J., Droogmans, G., \& Voets, T. (2005). Gating of TRP channels: a voltage connection? The Journal of Physiology, 567(1), 35-44. doi:10.1113/jphysiol.2005.088377

Ramachandran, P., \& Varoquaux, G. (2011). Mayavi: 3D Visualization of Scientific Data. Computing in Science \& Engineering, 13(2), 40-51. doi:10.1109/MCSE.2011.35 
bioRxiv preprint doi: $\mathrm{https}$ ://doi. org/10.1101/2020.07.16.207126; this version posted July 17, 2020. The copyright holder for this preprint (which was not certified by peer review) is the author/funder, who has granted bioRxiv a license to display the preprint in perpetuity. It is made available under aCC-BY 4.0 International license.

Romero, M. C., Davare, M., Armendariz, M., \& Janssen, P. (2019). Neural effects of transcranial magnetic stimulation at the single-cell level. Nature Communications, 10(1), 2642. doi:10.1038/s41467-019-10638-7

Stanley, S. A., Gagner, J. E., Damanpour, S., Yoshida, M., Dordick, J. S., \& Friedman, J. M. (2012). Radio-Wave Heating of Iron Oxide Nanoparticles Can Regulate Plasma Glucose in Mice. Science, 336(6081), 604-608. doi:10.1126/science.1216753

Stanley, S. A., Kelly, L., Latcha, K. N., Schmidt, S. F., Yu, X., Nectow, A. R., . . Friedman, J. M. (2016). Bidirectional electromagnetic control of the hypothalamus regulates feeding and metabolism. Nature, 531(7596), 647-650. doi:10.1038/nature17183

Stanley, S. A., Sauer, J., Kane, R. S., Dordick, J. S., \& Friedman, J. M. (2015). Remote regulation of glucose homeostasis in mice using genetically encoded nanoparticles. Nature Medicine, 21(1), 92-98. doi:10.1038/nm.3730

Usov, N. A. (2010). Low frequency hysteresis loops of superparamagnetic nanoparticles with uniaxial anisotropy. Journal of Applied Physics, 107(12), 123909. doi:10.1063/1.3445879

Wernsdorfer, W., Orozco, E. B., Hasselbach, K., Benoit, A., Barbara, B., Demoncy, N., . . Mailly, D. (1997). Experimental Evidence of the Néel-Brown Model of Magnetization Reversal. Physical Review Letters, 78(9), 1791-1794. doi:10.1103/PhysRevLett.78.1791

Wheeler, M. A., Deppmann, C. D., Patel, M. K., \& Güler, A. D. (2019). Reply to: Magneto is ineffective in controlling electrical properties of cerebellar Purkinje cells, Assessing the utility of Magneto to control neuronal excitability in the somatosensory cortex and Revaluation of magnetic properties of Magneto. Nature Neuroscience. doi:10.1038/s41593-019-0472-6

Wheeler, M. A., Smith, C. J., Ottolini, M., Barker, B. S., Purohit, A. M., Grippo, R. M., . . Güler, A. D. (2016). Genetically targeted magnetic control of the nervous system. Nature Neuroscience, 19(5), 756-761. doi:10.1038/nn.4265 